\title{
STEADY-STATE HEAT TRANSFER IN A PLATE WITH TEMPERATURE-DEPENDENT THERMAL CONDUCTIVITY (AN EXACT SOLUTION)
}

\author{
A. Miguelis ${ }^{\mathrm{a}}$, \\ R. Pazetto ${ }^{b}$, \\ and R. M. S. Gama \\ ${ }^{\text {a}}$ Universidade do Estado do Rio de Janeiro \\ Departamento de Engenharia Mecânica \\ Rua São Francisco Xavier 524 \\ CEP: 20550-900, Rio de Janeiro, RJ, Brazil \\ alane.miguelis@gmail.com \\ rsgama@terra.com.br

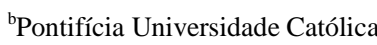 \\ do Rio de Janeiro \\ Departamento de Engenharia de Materiais \\ Rua Marquês de São Vicente 225 \\ CEP: 22451-900, Rio de Janeiro, RJ, Brazil \\ rogpaz1998@gmail.com \\ Received: November 10, 2018 \\ Revised: November 23, 2018 \\ Accepted: February 12, 2019
}

\section{ABSTRACT}

This work presents the solution of the steady-state heat transfer problem in a rectangular plate with an internal heat source in a context in which the thermal conductivity depends on the local temperature. This generalization of one of the most classical heat transfer problems is carried out with the aid of the Kirchhoff transformation and employs only well known tools, as the superposition of solutions and the Fourier series. The obtained results illustrate how the usual procedures may be extended for solving more realistic physical problems (since the thermal conductivity of any material is temperature-dependent). A general formula for evaluating the Kirchhoff transformation as well as its inverse is presented too. This work has a strong didactical contribution since such analytical solutions are not found in any classical heat transfer book. In addition, the main idea can be used in a lot of similar problems.

Keywords: nonlinear heat transfer; exact solution; Kirchhoff transformation

\section{NOMENCLATURE}

$H \quad$ plate width

$L \quad$ plate length

$k \quad$ thermal conductivity

$k_{R E F}$ thermal conductivity of reference

$\tilde{k}(T)$ thermal conductivity regarded as a function of the temperature

$\dot{q} \quad$ internal heat supply

$T$ temperature

$x, y \quad$ cartesian coordinates

\section{Greek symbols}

$\omega \quad$ Kirchhoff transformation

\section{INTRODUCTION}

One of the most studied partial differential equation is the one that represents the steady-state conduction heat transfer process in a body with constant thermal conductivity. In particular, for a two dimensional rectangular domain, this equation is mathematically represented as follows (Holman, J. P., 1996; Bergman, T. L., Lavine A. S., Incropera, F. P. and DeWitt, D. P., 2011).

$$
k\left(\frac{\partial^{2} T}{\partial x^{2}}+\frac{\partial^{2} T}{\partial y^{2}}\right)+\dot{q}=0 \quad 0<x<L, 0<y<H
$$

in which $k$ represents the (constant) thermal conductivity and $\dot{q}$ represents the internal heat supply (a known function $\dot{q}=\hat{q}(x, y)$ ).

When Eq. (1) is subjected to Dirichlet boundary conditions (John, F., 1978), we have the following problem (a conduction heat transfer problem)

$$
\begin{aligned}
& k\left(\frac{\partial^{2} T}{\partial x^{2}}+\frac{\partial^{2} T}{\partial y^{2}}\right)+\dot{q}=0 \quad 0<x<L, 0<y<H \\
& T=f_{1}(y), \quad x=0,0<y<H \\
& T=f_{2}(y), \quad x=L, 0<y<H \\
& T=f_{3}(x), \quad 0<x<L, y=0 \\
& T=f_{4}(x), \quad 0<x<L, y=H
\end{aligned}
$$

whose solution is easily obtained as (Wylie, C. R., 1975)

$$
T=\Psi+\Phi
$$


in which $\Psi$ is any function such that (there exists infinitely many choices for $\Psi$ )

$$
\begin{aligned}
& \left(\frac{\partial^{2} \Psi}{\partial x^{2}}+\frac{\partial^{2} \Psi}{\partial y^{2}}\right)= \\
& -\frac{\dot{q}}{k} \quad 0<x<L, 0<y<H, \quad \Psi=\hat{\Psi}(x, y)
\end{aligned}
$$

For instance, if $\dot{q}=$ constant , $\Psi$ may be given as

$$
\Psi=-\frac{\dot{q}}{4 k}\left(x^{2}+y^{2}\right)
$$

So, the function $\Phi$ must be such that

$$
\begin{aligned}
& \left(\frac{\partial^{2} \Phi}{\partial x^{2}}+\frac{\partial^{2} \Phi}{\partial y^{2}}\right)=0 \quad 0<x<L, 0<y<H \\
& \Phi=f_{1}(y)-\hat{\Psi}(0, y), \quad x=0,0<y<H \\
& \Phi=f_{2}(y)-\hat{\Psi}(L, y), \quad x=L, 0<y<H \\
& \Phi=f_{3}(x)-\hat{\Psi}(x, 0), \quad 0<x<L, y=0 \\
& \Phi=f_{4}(x)-\hat{\Psi}(x, H), \quad 0<x<L, y=H
\end{aligned}
$$

The function $\Phi$ may be represented as the following sum

$$
\Phi=\phi_{1}+\phi_{2}+\phi_{3}+\phi_{4}
$$

in which

$$
\begin{aligned}
& \left(\frac{\partial^{2} \phi_{1}}{\partial x^{2}}+\frac{\partial^{2} \phi_{1}}{\partial y^{2}}\right)=0 \quad 0<x<L, 0<y<H \\
& \phi_{1}=f_{1}(y)-\hat{\Psi}(0, y), \quad x=0,0<y<H \\
& \phi_{1}=0, \quad x=L, 0<y<H \\
& \phi_{1}=0, \quad 0<x<L, y=0 \\
& \phi_{1}=0, \quad 0<x<L, y=H
\end{aligned}
$$$$
\left(\frac{\partial^{2} \phi_{2}}{\partial x^{2}}+\frac{\partial^{2} \phi_{2}}{\partial y^{2}}\right)=0 \quad 0<x<L, 0<y<H
$$$$
\phi_{2}=0, \quad x=0,0<y<H
$$$$
\phi_{2}=f_{2}(y)-\hat{\Psi}(L, y), \quad x=L, 0<y<H
$$$$
\phi_{2}=0, \quad 0<x<L, y=0
$$$$
\phi_{2}=0, \quad 0<x<L, y=H
$$$$
\left(\frac{\partial^{2} \phi_{3}}{\partial x^{2}}+\frac{\partial^{2} \phi_{3}}{\partial y^{2}}\right)=0 \quad 0<x<L, 0<y<H
$$$$
\phi_{3}=0, \quad x=0,0<y<H
$$$$
\phi_{3}=0, \quad x=L, 0<y<H
$$

$$
\begin{aligned}
& \phi_{3}=f_{3}(x)-\hat{\Psi}(x, 0), \quad 0<x<L, y=0 \\
& \phi_{3}=0, \quad 0<x<L, y=H \\
& \left(\frac{\partial^{2} \phi_{4}}{\partial x^{2}}+\frac{\partial^{2} \phi_{4}}{\partial y^{2}}\right)=0 \quad 0<x<L, 0<y<H \\
& \phi_{4}=0, \quad x=0,0<y<H \\
& \phi_{4}=0, \quad x=L, 0<y<H \\
& \phi_{4}=0, \quad 0<x<L, y=0 \\
& \phi_{4}=f_{4}(x)-\hat{\Psi}(x, H), \quad 0<x<L, y=H
\end{aligned}
$$

The functions $\phi_{1}, \phi_{2}, \phi_{3}$ and $\phi_{4}$ are, therefore, given by (Carslaw, H. S. and Jaeger, J. C., 1959 )

$$
\begin{gathered}
\phi_{1}=\sum_{n=1}^{\infty} A_{n} \operatorname{senh}\left(\frac{n \pi(L-x)}{H}\right) \operatorname{sen}\left(\frac{n \pi y}{H}\right), \text { with } \\
A_{n}=\frac{2 \int_{0}^{H}\left\{f_{1}(y)-\hat{\Psi}(0, y)\right\} \operatorname{sen}\left(\frac{n \pi y}{H}\right) d y}{H \operatorname{senh}\left(\frac{n \pi L}{H}\right)}
\end{gathered}
$$

$\phi_{2}=\sum_{n=1}^{\infty} A_{n} \operatorname{senh}\left(\frac{n \pi x}{H}\right) \operatorname{sen}\left(\frac{n \pi y}{H}\right)$, with

$$
A_{n}=\frac{2 \int_{0}^{H}\left\{f_{2}(y)-\hat{\Psi}(L, y)\right\} \operatorname{sen}\left(\frac{n \pi y}{H}\right) d y}{H \operatorname{senh}\left(\frac{n \pi L}{H}\right)}
$$

$$
\begin{gathered}
\phi_{3}=\sum_{n=1}^{\infty} A_{n} \operatorname{senh}\left(\frac{n \pi(H-y)}{L}\right) \operatorname{sen}\left(\frac{n \pi x}{L}\right), \text { with } \\
A_{n}=\frac{2 \int_{0}^{L}\left\{f_{3}(x)-\hat{\Psi}(x, 0)\right\} \operatorname{sen}\left(\frac{n \pi x}{L}\right) d x}{L \operatorname{senh}\left(\frac{n \pi H}{L}\right)}
\end{gathered}
$$

$$
\begin{gathered}
\phi_{4}=\sum_{n=1}^{\infty} A_{n} \operatorname{senh}\left(\frac{n \pi y}{L}\right) \operatorname{sen}\left(\frac{n \pi x}{L}\right), \text { with } \\
A_{n}=\frac{2 \int_{0}^{L}\left\{f_{4}(x)-\hat{\Psi}(x, H)\right\} \operatorname{sen}\left(\frac{n \pi x}{L}\right) d x}{L \operatorname{senh}\left(\frac{n \pi H}{L}\right)}
\end{gathered}
$$

\section{TEMPERATURE-DEPENDENT THERMAL CONDUCTIVITY}

It is remarkable that the thermal conductivity of any real material depends on the temperature (Goldberg, et al., 2001; Glassbrenner and Slack, 1964; Gama, 2014). 
Now, let us consider the steady-state conduction heat transfer problem with temperature-dependent thermal conductivity. In this case, instead of Eq. (2), we will have the following nonlinear problem (here $k=\tilde{k}(T))$

$$
\begin{aligned}
& \frac{\partial}{\partial x}\left(k \frac{\partial T}{\partial x}\right)+\frac{\partial}{\partial y}\left(k \frac{\partial T}{\partial y}\right)+\dot{q}=0 \\
& 0<x<L, 0<y<H \\
& T=f_{1}(y), \quad x=0,0<y<H \\
& T=f_{2}(y), \quad x=L, 0<y<H \\
& T=f_{3}(x), \quad 0<x<L, y=0 \\
& T=f_{4}(x), \quad 0<x<L, y=H
\end{aligned}
$$

Problem (13) can not be solved with the tools used for solving (2).

So, aiming to construct the exact solution of (13), let us introduce the Kirchhoff transformation as follows (Arpaci, V. S., 1966)

$$
\omega=\frac{1}{k_{R E F}} \int_{0}^{T} \hat{k}(\xi) d \xi
$$

in which $k_{R E F}$ is a positive constant (in general the thermal conductivity at a given temperature). From the Kirchhoff transformation we have that

$$
\begin{aligned}
& \frac{\partial \omega}{\partial x}=\frac{\partial}{\partial x}\left(\frac{1}{k_{R E F}} \int_{0}^{T} \hat{k}(\xi) d \xi\right)=\frac{\tilde{k}(T)}{k_{R E F}} \frac{\partial T}{\partial x} \\
& \frac{\partial \omega}{\partial y}=\frac{\partial}{\partial y}\left(\frac{1}{k_{R E F}} \int_{0}^{T} \hat{k}(\xi) d \xi\right)=\frac{\tilde{k}(T)}{k_{R E F}} \frac{\partial T}{\partial y}
\end{aligned}
$$

and, therefore, problem (13) yields

$$
\begin{aligned}
& k_{R E F}\left(\frac{\partial^{2} \omega}{\partial x^{2}}+\frac{\partial^{2} \omega}{\partial y^{2}}\right)+\dot{q}=0 \quad 0<x<L, 0<y<H \\
& \omega=F_{1}(y)=\frac{1}{k_{R E F}} \int_{0}^{f_{1}(y)} \hat{k}(\xi) d \xi, \quad x=0,0<y<H \\
& \omega=F_{2}(y)=\frac{1}{k_{R E F}} \int_{0}^{f_{2}(y)} \hat{k}(\xi) d \xi, \quad x=L, 0<y<H \\
& \omega=F_{3}(x)=\frac{1}{k_{R E F}} \int_{0}^{f_{3}(x)} \hat{k}(\xi) d \xi, \quad 0<x<L, y=0 \\
& \omega=F_{4}(x)=\frac{1}{k_{R E F}} \int_{0}^{f_{4}(x)} \hat{k}(\xi) d \xi, \quad 0<x<L, y=H
\end{aligned}
$$

It is easy to see that the structure of Eq.(16) is exactly the one of Eq.(2). Hence, the procedure for solving Eq. (2) can be used for solving Eq.(16).

Thus, the solution of Eq. (16) is given by the following equation (similar to Eq. (3))

$$
\omega=\Psi+\Phi
$$

One more time, $\Psi$ is any function such that

$$
\begin{aligned}
& \left(\frac{\partial^{2} \Psi}{\partial x^{2}}+\frac{\partial^{2} \Psi}{\partial y^{2}}\right)=-\frac{\dot{q}}{k_{R E F}} \quad 0<x<L, 0<y<H, \\
& \Psi=\hat{\Psi}(x, y)
\end{aligned}
$$

The function $\Phi$ may be, again, represented as in Eq. (7). In this case the functions $\phi_{1}, \phi_{2}, \phi_{3}$ and $\phi_{4}$ are given by

$$
\begin{gathered}
\phi_{1}=\sum_{n=1}^{\infty} A_{n} \operatorname{senh}\left(\frac{n \pi(L-x)}{H}\right) \operatorname{sen}\left(\frac{n \pi y}{H}\right), \text { with } \\
A_{n}=\frac{2 \int_{0}^{H}\left\{F_{1}(y)-\hat{\Psi}(0, y)\right\} \operatorname{sen}\left(\frac{n \pi y}{H}\right) d y}{H \operatorname{senh}\left(\frac{n \pi L}{H}\right)} \\
\phi_{2}=\sum_{n=1}^{\infty} A_{n} \operatorname{senh}\left(\frac{n \pi x}{H}\right) \operatorname{sen}\left(\frac{n \pi y}{H}\right), \quad \text { with } \\
A_{n}=\frac{2 \int_{0}^{H}\left\{F_{2}(y)-\hat{\Psi}(L, y)\right\} \operatorname{sen}\left(\frac{n \pi y}{H}\right) d y}{H \operatorname{senh}\left(\frac{n \pi L}{H}\right)} \\
\phi_{3}=\sum_{n=1}^{\infty} A_{n} \operatorname{senh}\left(\frac{n \pi(H-y)}{L}\right) \operatorname{sen}\left(\frac{n \pi x}{L}\right), \quad \text { with } \\
A_{n}=\frac{2 \int_{0}^{L}\left\{F_{4}(x)-\hat{\Psi}(x, H)\right\} \operatorname{sen}\left(\frac{n \pi x}{L}\right) d x}{2 \int_{0}^{L}\left\{F_{3}(x)-\hat{\Psi}(x, 0)\right\} \operatorname{sen}\left(\frac{n \pi x}{L}\right) d x} \\
A_{n}=\frac{\operatorname{senh}\left(\frac{n \pi y}{L}\right) \operatorname{sen}\left(\frac{n \pi x}{L}\right), \quad \text { with }}{L \operatorname{senh}\left(\frac{n \pi H}{L}\right)}
\end{gathered}
$$

and, so, we have the exact solution of Eq.(16).

\section{OBTAINING T FROM $\omega$}

Since the thermal conductivity is an always positive valued function with a positive lower bound, the inverse of the Kirchhoff transformation is ensured. For instance, if we assume that the thermal conductivity is a piecewise constant function of the temperature as follows 


$$
\begin{aligned}
& k=\tilde{k}(T)=\left\{\begin{array}{c}
k_{1} \text { for } \quad T \leq T_{1} \\
k_{i} \text { for } \quad T_{i} \geq T>T_{i-1}, \\
k_{N} \text { for } \quad T>T_{N-1}
\end{array}\right. \\
& \text { for } i=2,3,4, \ldots, N-1
\end{aligned}
$$

we have

$$
\begin{aligned}
& \omega=\frac{1}{k_{R E F}} \int_{0}^{T} \hat{k}(\xi) d \xi= \\
& \int \frac{1}{k_{R E F}}\left\{k_{1} T\right\} \text {, if } T \leq T_{1} \\
& \frac{1}{k_{R E F}}\left\{k_{2}\left(T-T_{1}\right)+k_{1} T_{1}\right\}, \text { if } T_{2} \geq T>T_{1} \\
& \left\{\frac{1}{k_{\text {REF }}}\left\{k_{3}\left(T-T_{2}\right)+k_{2}\left(T_{2}-T_{1}\right)+k_{1} T_{1}\right\} \text {, if } T_{3} \geq T>T_{2}\right. \\
& \frac{1}{k_{R E F}}\left\{k_{N}\left(T-T_{N-1}\right)+k_{N-1}\left(T_{N-1}-T_{N-2}\right)+\right. \\
& \left.\ldots+k_{2}\left(T_{2}-T_{1}\right)+k_{1} T_{1} \text {, if } T>T_{N-1}\right\}
\end{aligned}
$$

So, the inverse is explicitly given by

$$
\begin{aligned}
T=\frac{k_{R E F}}{2} & \left\{\left(\frac{1}{k_{1}}+\frac{1}{k_{N}}\right) \omega+\right. \\
+ & \left.\sum_{i=2}^{N}\left(\frac{1}{k_{i}}-\frac{1}{k_{i-1}}\right)\left(\left|\omega-\omega_{i-1}\right|-\omega_{i-1}\right)\right\}
\end{aligned}
$$

\section{CONCLUSIONS}

The steady-state conduction heat transfer problem in a heated plate consists of a phenomenon described in any textbook of heat transfer. Nevertheless, all the presented solutions assume that the thermal conductivity is a constant.

In this work, with the aid of very simple tools, we have shown the solution of problems in which the thermal conductivity depends on the local temperature, as it is observed for any material.

This work presents a new contribution for the spectrum of problems with available analytical solutions.

\section{ACKNOWLEDGEMENTS}

The author R. M. S. Gama gratefully acknowledge the financial support provided by the Brazilian agency CNPq.

\section{REFERENCES}

Arpaci, V. S., 1966, Conduction Heat Transfer, Addison-Wesley.

Bergman, T. L., Lavine, A. S., Incropera, F. P., and DeWitt, D. P., 2011, Fundamentals of Heat and Mass Transfer, 7th Edition, Wiley.
Carslaw, H. S., and Jaeger, J. C., 1959, Conduction of Heat in Solids, 2nd Edition, Oxford Press.

Gama, R. M. S., 2014, A Note on the Constant Thermal Conductivity Hypothesis and its Consequence for Conduction Heat Transfer Problems, Thermal Engineering, Vol. 13, pp. 48-51.

Glassbrenner, C. J., and Slack, G. A., 1964, Thermal Conductivity of Silicon and Germanium from $3^{\circ} \mathrm{K}$ to the Melting Point, Published by the American Physical Society, Vol. 134 , pp. 10581069.

Goldberg, Yu., Levinshtein, M. E., and Rumyantsev, S. L., 2001, Properties of Advanced Semiconductor Materials GaN, AlN, SiC, BN, SiC, SiGe, John Wiley \& Sons.

Holman, J. P., 1996, Heat Transfer, McGrawHill College.

John, F., 1978, Partial Differential Equations, 3rd Edition, Springer-Verlag.

Wylie, C. R., 1975, Advanced Engineering Mathematics, 4th Edition, McGraw-Hill. 